\title{
Dyadic Adjustment and Sense of Mastery of Pregnant Women Protect Against Prenatal Depression
}

\author{
Sung Hee Lee ${ }^{1}$ and Seung A Lee ${ }^{2} *$ \\ ${ }^{1}$ College of Nursing, Kyungpook National University, Daegu, KOREA \\ ${ }^{2}$ Doctoral student, College of Nursing, Kyungpook National University, \\ Daegu, KOREA \\ ${ }^{1}$ leesh@knu.ac.kr, 2angelnurse35@hanmail.net
}

\begin{abstract}
This study explored protective factors against prenatal depression in pregnant women. One-hundred thirty-four (134) pregnant women were recruited from an obstetric clinic and public health center in Korea. Dyadic adjustment, sense of mastery, and prenatal depression were assessed using structured questionnaires. Prenatal depression was assessed with the Edinburgh Postnatal Depression Scale (EDPS). Binomial logistic analysis was used to identify predictors of prenatal depression. Of the participants, $40.3 \%$ screened as positive on the EPDS with a cut-off point of 9/10. Protective factors against prenatal depression were a history of childbirth, dyadic adjustment, and sense of mastery. We must consider these findings when developing interventions for pregnant women focusing on prevention and management of prenatal depression.
\end{abstract}

Keywords: Dyadic adjustment, Sense of mastery, Prenatal depression, Pregnant women

\section{Introduction}

\subsection{Background}

Pregnancy and childbirth are major events that cause stress among women. Women going through pregnancy may experience emotional, social, and role changes as well as various physical changes [1]. In particular, a sudden change in hormone balance can cause physical discomfort, such as morning sickness and weight increase. Moreover, the reduced quality of sleep among other risk factors might increase the probability of depression among pregnant women [2].

According to studies of Western populations, about 5-15\% of expectant mothers experience prenatal depression [3]. In contrast, in Korea, about 12-36\% of expectant mothers experience prenatal depression [4-6]. Pregnant women suffering from prenatal depression are 5-6 times more likely than normal pregnant women to develop postnatal depression [7]. Furthermore, prenatal depression is known to be a strong preceding factor of postnatal depression [7-9]. The importance of prenatal depression, however, remains relatively unstudied despite being a common condition, like postnatal depression [10].

*Corresponding Author: Seung A Lee

Doctoral student, College of Nursing, Kyungpook National University

Tel: +82-10-4276-0713

E-mail: angelnurse35@ hanmail.net 
Pregnant women suffering from prenatal depression tend to have less healthy babies. Premature birth, low birth-weight infants, and growth delay in the prenatal period, as well as excessive activity increase, have been observed for pregnant women who experienced depression [11]. Furthermore, depressive pregnant women are less interested in their infants, resulting in reduced mother-infant interaction [12]. By measuring depression at the $7^{\text {th }}$ month of pregnancy, pregnant women with a high level of depression have been found to have poor awareness of maternal-fetal relationship [13]. This leads to disruption in the formation of maternal-fetal attachment [14].

However, not all pregnant women suffer from prenatal depression. Mercer et al. [15] suggested that the relationship between the pregnant woman and her partner and sense of mastery are protective factors against prenatal depression [15]. They suggested a theoretical model in which pregnant women's prenatal depression is reduced when her relationship with her partner is better and her sense of mastery is greater [15]. Dyadic adjustment is an indicator of couples' ability to resolve problems and adapt to each other [16], and prenatal depression is higher for pregnant women with no satisfaction from partner support compared to those who found partner support to be satisfying $[4,17,18]$. Sense of mastery refers to the extent to which a person considers his/her life changes to be under his/her control [19]. Women with a high sense of mastery can easily control pregnancy-related stress, and therefore experience fewer negative emotions [20]. According to previous studies, pregnant women with a high sense of mastery report lower levels of depression [20,21].

As described above, this study investigated depression among pregnant women and potential protective factors against it. This study is important for developing a nursing intervention for pregnant women to give birth to healthy children and prevent prenatal depression. However, until recently, most previous studies have investigated prenatal depression in pregnant women with regard to prenatal depression from psychosocial factors [22] as well as clinical studies about depression symptoms during pregnancy [5]. Accordingly, it is difficult to find research demonstrating dyadic adjustment and sense of mastery as protective factors against prenatal depression.

This study will identify the prevalence of prenatal depression among pregnant women, find factors to prevent prenatal depression and find the needs to prevent and manage pregnant women's prenatal depression. In addition, this study suggests base line data for developing nursing interventions for preventing and managing pregnant women's prenatal depression.

\subsection{Purpose}

The purpose of this study was to explore predictors of prenatal depression in pregnant women. The specific objectives were as follows:

1.2.1. To examine the level of dyadic adjustment, sense of mastery, and prenatal depression in pregnant women.

1.2.2. To compare non-depressed and depressed groups according to general and obstetric variables, dyadic adjustment, and sense of mastery.

1.2.3. To verify the protective factors against prenatal depression in pregnant women. 


\section{Methods}

\subsection{Study Design}

This was a descriptive cross-sectional study that used self-report questionnaires.

\subsection{Participants}

Participants were 134 pregnant women who visited the obstetrics and gynecology outpatient centers at a university hospital in the city of $\mathrm{D}$, and a public health center located in the city of Y. Participants were included if they were pregnant women in their 20th through 38th week of pregnancy and they agreed to participate in the study. The required sample size for binomial logistic regression using a significance level $(\alpha)$ of $.05, .80$ statistical power $(1-\beta)$, and an effect size $(d)$ of .15 was calculated using $G^{*}$ Power 3.1. The calculated sample size was 119 , but 200 women were invited to participate to account for non-response rate. Six questionnaires with missing data were excluded from the analyses.

\subsection{Instruments}

Revised Dyadic Adjustment Scale (RDAS)

To measure dyadic adjustment, the Revised Dyadic Adjustment Scale (RDAS), originally developed by Spanier [23], supplemented and revised by Busby et al. [24], and adapted by Choi [25], was used. This tool consists of 14 questions with five questions on agreement on opinions, four on satisfaction with relationship, two on cohesiveness, and three on expression of affection. A Likert scale was used with 1 point allocated to "not always" and 6 points to "always" for each question. Total scores range from 14 to 84 , and reverse coding is used for negatively worded questions. Higher values indicate higher dyadic adjustment. Reliability (Cronbach's $\alpha$ ) of the tool at the time of development was .81-.90, and our study showed a value of .90 .

\section{Sense of Mastery Scale (SOMS)}

Sense of mastery was measured using Pearlin et al.'s Sense of Mastery Scale (SOMS) [19]. This scale was validated by the Korea Gerontological Forum [26]. The SOMS consists of seven items. Each item is scored on a 1-4 scale, with higher scores indicating a higher sense of mastery. Total scores range from 7 to 28 , and reverse coding is used for negatively worded questions. The internal consistency/reliability at Pearlin et al.'s study was .61-67, and in this study, it was .80 .

\section{Edinburgh Postnatal Depression Scale (EPDS)}

Prenatal depression was determined with a widely used instrument, the Edinburgh Postnatal Depression Scale (EPDS) [27]. The scale was validated by Han et al. in Korea [28]. The EPDS consists of 10 items relevant to depressed mood experienced in the past week. Each item is scored on a 0-3 scale, with higher scores indicating higher levels of depression. In Korea, there have been several validation studies of the EPDS in perinatal pregnant samples [5,7,10]. Previous studies in Korea $[5,29,30]$ found that sensitivity was $77 \%$ and specificity was $87 \%$ using a cut-off score of $9 / 10$. The present study used this cut-off score as the basis for classifying an individual as depressed or non-depressed. Scores of 9 or less indicate no depression, while scores of 10 or greater indicate depression. Cronbach's $\alpha$ in the present study was .82 .

\subsection{Ethical Approval}


The study protocol was approved by the Institutional Review Board at K National University in Korea (IRB 2014-0010). Informed written consent was taken from all the participants for voluntary participation.

\subsection{Data Collection}

Data collection for this study was conducted between August 23 and November 25 , 2014, with an agreement from chairs of two hospitals and cooperation from obstetrics and gynecology outpatient center. The researcher and two trained assistants conducted the survey, and approximately 15 minutes were spent to finish a questionnaire.

\subsection{Statistical Analysis}

Data were analyzed with SPSS (version 20; IBM Corporation: Armonk, NY, USA). Descriptive statistics (frequencies, means, and standard deviations) were used for the general and obstetric characteristics and major variables in this study. Participants were divided into two groups based on their EPDS scores (depressed: greater than 10; non-depressed: 9 or less). The chi-squared test was applied to capture differences in the proportion of prenatal depression across sociodemographic and obstetrics variables. Binomial group comparisons were performed using t-tests for major variables. Binomial logistic regression was then performed, including variables that had a significant binomial relationship with the EPDS scores and across socio-demographic and obstetrics variables to verify protective factors against prenatal depression in pregnant women.

\section{Result}

\subsection{Participant Characteristics and Comparison of Non-Depressed and Depressed Groups on General and Obstetrics Characteristics}

Almost all subjects (62.0\%) were in their 30s. Most (80.6\%) had completed college or beyond, and $41 \%$ did not have an occupation. Monthly income ranging from $2,000,000$ to $4,000,000$ won made up $56.0 \%$, representing the highest percentage. More than half of the sample (59.7\%) was 27 weeks pregnant or less. Pregnant women without a history of childbirth accounted for $40.3 \%$, and those who planned pregnancy accounted for $87.3 \%$ (Table 1 ).

The overall prevalence of prenatal depression, as defined by EPDS scores of 10 or greater, was $40.3 \%$. Univariate analysis revealed no significant association of socio-demographic and obstetric variables with prenatal depression (Table 1).

Table 1. General and Obstetric Variables and Comparison on NonDepressed and Depressed Groups on General and Obstetric Variables $(N=134)$

\begin{tabular}{|c|c|c|c|c|c|}
\hline \multirow{3}{*}{ Variables } & \multirow{2}{*}{ Total } & $\begin{array}{c}\text { Non- } \\
\text { depressed }\end{array}$ & Depressed & \multirow{3}{*}{$\chi^{2}$} & \multirow{3}{*}{$p$} \\
\hline & & $\begin{array}{l}(\mathrm{n}=80, \\
59.7 \%)\end{array}$ & $\begin{array}{l}(\mathrm{n}=54, \\
40.3 \%)\end{array}$ & & \\
\hline & $\mathrm{n}(\%)$ & $\mathrm{n}(\%)$ & $\mathrm{n}(\%)$ & & \\
\hline \multirow{2}{*}{ Age } & $\leq 30$ & $56(41.8)$ & $33(41.2)$ & $23(42.6)$ & \multirow{2}{*}{.024} \\
\hline & $\geq 31$ & $78(58.2)$ & $47(58.8)$ & $31(57.4)$ & \\
\hline \multirow{2}{*}{ Education } & $\leq$ High school & $26(19.4)$ & $13(16.3)$ & $13(24.1)$ & \multirow{2}{*}{1.262} \\
\hline & $\geq$ College & $108(80.6)$ & $67(83.8)$ & $41(75.9)$ & \\
\hline
\end{tabular}




\begin{tabular}{clcccc} 
Occupation & Yes & $55(41.0)$ & $31(38.8)$ & $24(44.4)$ & \multirow{2}{*}{.432} \\
& No & $79(59.0)$ & $49(61.3)$ & $30(55.0)$ & \\
Family income & $<200$ & $14(10.4)$ & $7(8.8)$ & $7(13.0)$ & \\
$(1000$ won $)$ & $200-400$ & $75(56.0)$ & $44(55.0)$ & $31(57.4)$ & 1.002 \\
& $\geq 401$ & $45(33.6)$ & $29(36.3)$ & $16(29.6)$ & \\
Gestational age & $\leq 27$ & $80(59.7)$ & $22(27.5)$ & $17(31.5)$ & .248 \\
& $\geq 28$ & $54(40.3)$ & $58(72.5)$ & $37(68.5)$ & \\
History of & 0 & $54(40.3)$ & $28(35.0)$ & $26(48.1)$ & \\
childbirth & 1 & $59(44.0)$ & $36(45.0)$ & $23(42.6)$ & 3.799 \\
& 2 & $21(15.7)$ & $16(20.0)$ & $5(9.3)$ & \\
Wanted pregnancy & Yes & $117(87.3)$ & $70(87.5)$ & $47(87.0)$ & .006 \\
& No & $17(12.7)$ & $10(12.5)$ & $7(13.0)$ & \\
\hline
\end{tabular}

RDAS: Revised Dyadic Adjustment Scale; SOMS: Sense of Mastery Scale.

\subsection{RDAS and SOMS and Comparison of Non-Depressed and Depressed Groups}

The mean RDAS score was 63.9 (SD $=11.70)$, and the mean SOMS score was 20.2 (SD = 3.03). There were significant differences between non-depressed and depressed groups in the scores for the RDAS $(\mathrm{t}=3.661, p<.001)$ and SOMS $(\mathrm{t}=$ $4.475, p<.001)$ (Table 2).

Table 2. RDAS and SOMS and Comparison of Non-Depression and Depression Groups on RDAS and SOMS ( $N=134)$

\begin{tabular}{|c|c|c|c|c|c|}
\hline Variables & Total & $\begin{array}{c}\text { Non- } \\
\text { depressed } \\
(\mathrm{n}=80)\end{array}$ & $\begin{array}{c}\text { Depressed } \\
(\mathrm{n}=54)\end{array}$ & $\mathrm{t}$ & $p$ \\
\hline & $\mathrm{M} \pm \mathrm{SD}$ & $\mathrm{M} \pm \mathrm{SD}$ & $\mathrm{M} \pm \mathrm{SD}$ & & \\
\hline RDAS & $\begin{array}{l}63.9 \pm \\
11.70\end{array}$ & $\begin{array}{l}66.85 \pm \\
11.07\end{array}$ & $\begin{array}{l}59.63 \pm \\
11.39\end{array}$ & 3.661 & $\begin{array}{r}< \\
.001\end{array}$ \\
\hline SOMS & $20.2 \pm 3.03$ & $\begin{array}{l}21.15 \pm \\
3.04\end{array}$ & $\begin{array}{l}16.80 \pm \\
2.44\end{array}$ & 4.745 & $\begin{array}{r}< \\
.001\end{array}$ \\
\hline
\end{tabular}

\subsection{Predictors of Prenatal Depression According to Binomial Logistic Regression in Unmarried Pregnant Women}

To determine the factors protecting prenatal depression in pregnant women, all the general and obstetric variables, RDAS, and SOMS were introduced as independent variables in the binomial logistic regression model. Results of the binomial logistic regression are presented in Table 3. A higher risk for prenatal depression was associated with a history of nulliparity $(\operatorname{Exp}(B)=5.51, p=.016)$, lower RDAS $(\operatorname{Exp}(\mathrm{B})=.95, p=.017$, and lower SOMS $(\operatorname{Exp}(\mathrm{B})=.75, p=.001)$. No other variables were statistically significant in the regression model. Therefore, a history of childbirth, dyadic adjustment, and sense of mastery protected against prenatal depression in pregnant women (Table 3).

Table 3. Predictors of Prenatal Depression According to Binomial Logistic Regression in Pregnant Women ( $N=134)$

\begin{tabular}{cccc}
\hline Variables & $\begin{array}{c}\text { Adjusted } \\
\text { Odds }\end{array}$ & $\begin{array}{c}95 \% \\
\text { Confidence }\end{array}$ & $p$ \\
\hline
\end{tabular}




\begin{tabular}{|c|c|c|c|c|}
\hline \multirow{2}{*}{ Age (years; Reference: $\geq 31$ ) } & \multirow[t]{2}{*}{ Ratio } & \multicolumn{2}{|c|}{ Interval } & \multirow[b]{3}{*}{.636} \\
\hline & & & & \\
\hline$\leq 30$ & 1.23 & .52 & 2.93 & \\
\hline Education (Reference: $\geq$ College) & & & & \\
\hline$\leq$ High school & .92 & .34 & 2.51 & .875 \\
\hline Occupation (Reference: No) & & & & \\
\hline Yes. & 1.15 & .47 & 2.85 & .756 \\
\hline Family income $(\geq 401)$ & & & & \\
\hline$<200$ & 1.89 & .47 & 7.56 & .368 \\
\hline $200-400$ & 1.81 & .71 & 4.67 & .217 \\
\hline Gestational age(Reference: $\geq 28)$ & & & & \\
\hline $\begin{array}{l}\leq 27 \\
\text { History of childbirth(Reference: } \geq 2 \text { ) }\end{array}$ & 1.20 & .48 & 2.95 & .699 \\
\hline 0 & 5.51 & 1.37 & 22.22 & .016 \\
\hline 1 & 4.07 & 1.05 & 15.74 & .042 \\
\hline Wanted pregnancy (Reference: No) & & & & \\
\hline Yes & .95 & .27 & 3.31 & .929 \\
\hline RDAS & .95 & .92 & .99 & .017 \\
\hline SOMS & .75 & .62 & .89 & .001 \\
\hline
\end{tabular}

RDAS: Revised Dyadic Adjustment Scale; SOMS: Sense of Mastery Scale.

\section{Discussion}

To understand factors that protect the pregnant women against prenatal depression, a cross-sectional survey was conducted on 134 pregnant women. The following paragraphs discuss the findings.

The overall prevalence of prenatal depression, as defined by EPDS scores of 10 or greater [29], was $40.3 \%$. According to other Korean studies using the same techniques (i.e., depression measurement tool and cut-off point 9/10), prenatal depression prevalence was $26.3 \%$ [5] and $28.9 \%$ [22]. Thus, our subjects showed a higher level of prenatal depression as compared to previous studies. Demographic variables, the measurement method, and time difference might have raised the rate of prenatal depression. There is a need for evaluating subjects' depression status through standardized interviews in future research. According to studies from Western cultures using the EPDS, 5.8\% of pregnant women suffer from both prenatal and postnatal depression [31], and 9\% of pregnant women suffer from just prenatal depression [32], showing lower prenatal depression incidence compared to Korea. Some countries already employ national policies because of the seriousness of prenatal depression among pregnant women. In the US, the government provides support for eligible women. This support concerns arranging pre/postnatal depression policies, systems, and services, and some states are already implementing these programs [33-34]. In the UK, prenatal management including depression assistance is already being implemented through local social public health projects [34]. However, in Korea, screening inspection for prenatal depression is not yet compulsory for pregnant women, and healthcare providers are not yet interested in prenatal depression management. Accordingly, the Korean government should include prenatal depression management in pregnant women support policies in order to provide intervention care for pregnant women in their early prenatal depression stage through health centers and obstetric and gynecology hospitals.

A history of childbirth, dyadic adjustment, and sense of mastery were protective factors against prenatal depression in pregnant women. Specifically, the more a woman experiences childbirth, the lower the risk of prenatal depression. These 
findings deviate from previous results proposing that pregnant women's birth experience does not have any effect on prenatal depression $[5,6,10,14,18]$, and that multiparity is more likely to lead to prenatal depression than is nulliparity [35]. Nulliparity might lead to an increase in pregnancy-induced stress because of physical, emotional, social, and maternal role changes in their first pregnancy [1]. Pregnancy-induced stress was a strong predictive factor of prenatal depression $[6,36]$. About $60 \%$ of the subjects have already experienced more than one birth, and they experienced less stress compared to nulliparous women. This finding suggests that birth experience plays an important role as a protective factor against prenatal depression among pregnant women. There is a need for replication studies measuring prenatal depression among pregnant women.

We found that the risk for pregnant women to suffer prenatal depression is lower when their dyadic adjustment level is high. This result supports previous studies proposing that a high level of marital adaptability, marital satisfaction, and couple intimacy can reduce depression in pregnant women [2,4,17,37]. Dyadic adjustment is closely related to couples' support status, such that as a partner puts in more support, their dyadic adjustment level increases [38]. It has also been reported that pregnant women's prenatal depression increases with reduced partner support. In particular, with decreases in support related to acts of respect, affection, trust, and interest, prenatal depression increases [39]. Therefore, it is crucial to help spouses of pregnant women to participate in prenatal education and allow them to realize that their support is very important in terms of managing prenatal depression. Healthcare providers including nurses should intervene with a patient's prenatal management with the understanding that prenatal depression and dyadic adjustment are correlated.

The risk of prenatal depression was low when sense of mastery was high. Sense of mastery is a belief that one can judge and implement behaviors, and so it reflects a specific intensity of confidence. This sense of mastery is directly affected by selfesteem, and thus, as self-esteem increases, so does sense of mastery [19]. Selfesteem is a strong factor that may trigger prenatal depression, and so many pregnant women with low self-esteem experience prenatal depression [6]. This study's result is identical to findings proposing that high self-esteem can bring about low pre- and postnatal depression [20]. Therefore, in order to assess and improve pregnant women's self-esteem and sense of mastery during prenatal management, intervention in nursing practice should be considered.

Dyadic adjustment and sense of mastery, as proposed in Mercer et al.'s theoretical models [15], have been identified as factors that may protect against prenatal depression. By this means, this study is significant because it verified the idea that high dyadic adjustment and sense of mastery can lead to a reduced risk of prenatal depression. However, there is a limitation in generalizing this research outcome to all pregnant women because the participants were chosen using convenience sampling.

\section{Conclusion}

A substantial proportion of pregnant women who came for a prenatal check-up to our study setting are at risk of prenatal depression, which was associated with a history of childbirth, dyadic adjustment, and sense of mastery. Therefore, healthcare providers including nurses need to screen for depression at every opportunity in the prenatal period. Furthermore, nursing interventions must focus on both dyadic adjustment and sense of mastery, as these might be effective for decreasing prenatal depression in pregnant women. Further studies are needed to develop such interventions and verify their effects. Future research may benefit from repeated 
studies including various predictors of prenatal depression in pregnant women, including partner support and pregnancy-induced stress.

\section{References}

[1] H. J. Lee, O. K. Hyun and J. H. Rha, "Longitudinal Study on the Changes of Mothers' Stress and Cortisol From Pregnancy to Postpartum Period", The Korean Society of Community Living Science, vol. 17, no. 3, (2006), pp. 109-121

[2] H. W. Kim, and Y. Y. J, "Influencing Factors on Antenatal Depression", Journal Korean Society of Women Health Nursing, vol. 16, no. 2, (2010), pp. 95-104.

[3] O. Chatillon and C. Even, "Antepartum Depression: Prevalence, Diagnosis and Treatment", L' Encephale, vol. 36, no. 6, (2010), pp. 443-451.

[4] J. H. Kwon, "A Test of a Vulnerability-Stress Model of Prepartum Depression", Korean Journal of Clinical Psychology, vol. 15, no. 1, (1996), pp. 33-43.

[5] S. K. Choi, Y. A. Ahn, J. C. Shin and D. G. Gyu, "A Clinical Study of Depressive Symptoms During Pregnancy", Korean Journal of Obstetrics and Gynecology, vol. 52, no. 11, (2009), pp. 1102-1108.

[6] Y. L. Kim and C. W. Chung, "Factors of Prenatal Depression by Stress-Vulnerability and Stress-Coping Model", Korean Journal of Women Health Nursing, vol. 20, no. 1, (2014), pp. 38-47.

[7] A. Bowen and N. Muhajarine, "Antepartum Depression", Canadian Nurse, vol. 102, no. 9, (2006), pp. 27-30.

[8] T. Kitamura, K. Yoshida, T. Okano, K. Kinoshita, M. Hayashi, N. Toyoda, M. Ito, N. kudo, K. Tada, K. Kanazawam, K. Sakumoto, S. Satoh, T. Furukawa and H. Nakano, "Multicentre Prospective Study of Prenatal Depression in Japan: Incidence and Correlates of Antenatal and Postnatal Depression", Archives of Women's Mental Health, vol. 9, no. 3, (2006), pp. 121-130.

[9] B. Leigh and J. Milgron, "Risk Factors for Antenatal Depression, Postnatal Depression and Parenting Stress", BioMed Central Psychiatry, vol. 8, no. 24, (2008), pp. 1-11.

[10] E. J. Lee and J. S. Park, "Status of Antepartum Depression and Its Influencing Factors in Pregnant Women", Journal of the Korea Academia-Industrial Cooperation Society, vol. 14, no. 8, (2013), pp. 3897-3906.

[11] T. Field, M. Diego, M. Hernandez-Reif, B. Figueiredo, S. Schanberg and C. Kuhn, "Sleep disturbance in Depressed Pregnant Women and Their Newborns", Infant Behavior and Development, vol. 30, no. 1, (2007), pp. 127-133.

[12] E. H. Moon, J. Y. Kim, M. K. Jeung, H. M. Son and J. N. Oh, “Anxiety-Depression and Maternal Fetal Attachment Behaviors of Pregnant Women with Preterm Labor and Normal Pregnant Women”, Journal The Academic Society of Parent-Child Health, vol. 9, no. 2, (2006), pp. 128-139.

[13] B. Figueiredo and R. Costa, "Mother's Stress, Mood and Emotional Involvement with the Infant: 3 months before and 3 months after childbirth", Archives of Women's Mental Health, vol. 12, no. 3, (2009), pp. 143-153.

[14] M. K. Kwon, "Antenatal Depression and Mother-Fetal Interaction", Journal of Korean Academy of Child Health Nursing, vol. 13, no. 4, (2007), pp. 416-426.

[15] R. T. Mercer, A. M. Katharyn, S. Ferketich and J. DeJoseph, "Theoretical Models for Studying the Effect of Antepartum Stress on the Family", Research in Nursing and Health, vol. 35, no. 6, (1986), pp. 339-346.

[16] H. J. Locke and K. M. Wallace, "Short Marital-Adjustment and Prediction Test: Their Reliability and Validity", Marriage and Family Living, vol. 21, no. 3, (1959), pp. 251-255.

[17] K. D. O'Leary, J. L. Christin and N. R. Mendell, "A Closer Look at the Link between Marital Discord and Depressive Symptomatology", Journal of Social and Clinical Psychology, vol. 14, (1994), pp. 1-9.

[18] H. Wee and S. Y. Park, "The Relationships between Anxiety, Depression, Prenatal Stress, MaternalFetal Attachment and Gratitude", Journal of Korean Society Maternal and Child Health, vol. 16, no. 2, (2012), pp. 274-286.

[19] L. I. Pearlin, M. A. Lieberman, E. G. Menaghan and J. T. Mullan, "The Stress Process", Journal of Health and Social Behavior, vol. 22, no. 4, (1981), pp. 337-356.

[20] Y. K. Shin and Y. S. Choi, "The Effect of Self Efficacy, Marital Conflicts and Prenatal Depression on Postpartum Depression”, Journal of Korean Association of Family Relations, vol. 18, no. 1, (2013), pp. $155-176$

[21] M. J. Seo, "The Relationship between a Father's Involvement in Parental Roles and Postpartum Depression: The Mediation Effects of Marital Relationships and the Moderating Effects of Mother's Self-Perception", Journal of Korean Association of Child Studies, vol. 32, no. 6, (2011), pp. 107-121.

[22] M. E. Lee and Y. R. Kweon, "Psycho-Social Factors Association with Depression in Pregnant Women", Journal of Korean Academy of Psychiatric Mental Health Nursing, vol. 20, no. 3, (2011), pp. 252-260.

[23] G. B. Spanier, "Measuring Dyadic Adjustment Scale: New Scales for Assessing the Quality of Marriage and Similar Dyads", Journal of Marriage and the Family, vol. 38, no. 1, (1976), pp. 15-28. 
[24] D. M. Busby, C. Christensen, D. R. Crane and J. H. Larson, "A Revision of the Dyadic Adjustment Scale for use with Distressed and Nondistressed Couple: Construct Hierarchy and Multidimensional Scales", Journal of Marital and Family Therapy, vol. 21, no. 3, (1995), pp. 289-308.

[25] H S. I. Choi, "Revision Dyadic Adjustment Scale: Reliability and Validity", Gho Hwang Non Jip Kyung Hee Graduate School, vol. 35, (2004).

[26] Korean Gerontology Forum, "Older Scale", House of Sharing, (2010).

[27] J. Cox, J. Holden and R. Sagovsky, "Detection of Postnatal Depression: Development of the 10-item Edinburgh Postnatal Depression Scale", The British Journal of Psychiatry, vol. 150, no. 6, (1987), pp. 782-786.

[28] K. W. Han, M. J. Kim and J. M. Park, "The Edinburgh Postnatal Depression Scale, Korean Version: Reliability and Validity", Journal of Korean Society of Biological Therapies in Psychiatry, vol. 10, no. 2, (2004), pp. 201-207.

[29] Y. K. Kim, J. W. Hur, K. H. Kim, K. S. Oh and Y. C. Shin, "Clinical Application of Korean Version of Edinburgh Postnatal Depression Scale", Journal of Korean NeuroPsychiatric Association, vol. 47, no. 1, (2008), pp. 36-44.

[30] C. S. Ahn, M. S. Kang, S. Y. Park and Y. R. Choi, "Usefulness of Edinburgh Postnatal Depression Scale for Postpartum Depression”, Korean Journal of Perinatology, vol. 26, no. 1, (2015), pp. 21-27.

[31] C. Rubertsson and U. Waldenstrom, "Depressive Mood in Early Pregnancy and Postpartum: Prevalence and Women at Risk in a National Swedish Sample", Journal of Reproductive and Infant Psychology, vol. 23, no. 2, (2005), pp. 155-166.

[32] J. W. Rich-Edwards, K. Kleinman, A. Abrams, B. L. Harlow, T. J. McLaughlin, H. Joffe and M. W. Gillman, "Sociodemographic Predictors of Antenatal and Postpartum Depressive Symptoms among Women in a Medical Group Practice", Journal of Epidemiology Community Health, vol. 60, no. 3, (2006), pp. 221-227.

[33] University Management center for health promotion, "The Study for Policy Plan on the Actual Depressed Condition of Perinatal and the Decrease of Incident Rate", (2010), pp. 33-37.

[34] N. M. Hwang, "The Necessity for Establishing a Public Prenatal Health Promotion and Education System”, Health Welf Policy Forum, vol. 163, (2006), pp. 50-64.

[35] H E. S. Kim and S. Y. Rue, "The Relationship between Depression and Sociopsychology Factors in Pregnant Women", Journal of The Korean Society of Maternal and Child Health, vol. 12, no. 2, (2008), pp. 228-241.

[36] M. K. Kwon and K. S. Bang, "Relationship of Prenatal Stress and Depression to Maternal-Fetal Attachment and Fetal Growth", Journal of Korean Academy of Nursing, vol. 41, no. 2, (2011), pp. 276283.

[37] J. E. Lee and N. W. Chung, "The Relationship of Family-of-Origin Differentiation and Prepartum Depression: The Mediation Effect of Couples' Marital Intimacy”, Journal of Family and Counseling, vol. 4, no. 1, (2014), pp. 19-34.

[38] S. H. Lee and S. A. Lee, "Protective Factors against Prenatal Depression in Pregnant Women", Advanced Science and Technology Letters, vol. 122 (IRTT 2016), pp. 78-82.

[39] H. J. Cho and J. H. Kwon, "A Test of the Attachment Model of Prepartum Depression”, The Korean Journal of Clinical Psychology, vol. 21, no. 4, (2002), pp. 727-744.

\section{Authors}

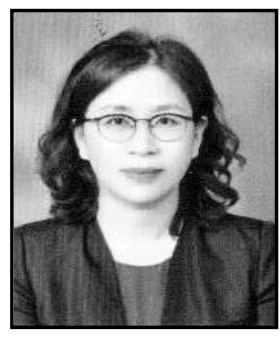

Sung Hee Lee, she is a professor of the College of Nursing at Kyungpook National University. She is an RN and has a Ph.D. degree in Nursing Science from Kyungpook National University. Her research interests are low fertility, fetal attachment behaviors and date violence.

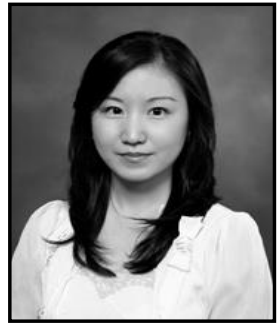

Seung A Lee, she is a student of the College of Nursing at Kyungpook National University. She is an RN and has a Master's degree in Nursing Science from Kyungpook National University. Her research interests are women's health nursing and Gynecologic Oncology. 
International Journal of Bio-Science and Bio-Technology vol. 8, No.3 (2016) 